\title{
Prostate Carcinosarcoma Developing After Combined Pelvic Extemal Beam Radiation Therapy and Androgen Deprivation Therapy
}

\author{
Young-Joo Kim
}

Department of Urology, Jeju National University Hospital, Jeju National University College of Medicine, Jeju, Korea

\begin{abstract}
Carcinosarcoma of the prostate is a very rare disease, which consists of carcinomatous and sarcomatous components. The prognosis of patients with prostatic carcinosarcoma is poor. An 80-year-old man who had been suffering from urinary retention and constipation with rectal pain visited a hospital. A patient with a history of combined pelvic external beam radiation therapy (EBRT) and androgen deprivation therapy (ADT) for prostate adenocarcinoma 7 years ago was diagnosed with secondary malignancy such as prostate carcinosarcoma by prostate biopsy. This is also a rare case occurring after combined EBRT and ADT for adenocarcinoma in a short interval. Then, he underwent chemotherapy for one cycle. Chemotherapy was stopped because of rapid clinical aggravation, and the patient died 5 months later. It is important to keep in mind the possibility of carcinosarcoma in the case that prostate-specific antigen maintains stable level but extent of tumor is gradually increased after EBRT or ADT. (Korean J Urol Oncol 2019;17:195-198)
\end{abstract}

Key Words: Prostate $\cdot$ Carcinosarcoma $\cdot$ Prognosis

Carcinosarcoma of prostate is extremely rare cancer developing after external beam radiation therapy (EBRT) or androgen deprivation therapy (ADT). Carcinosarcoma is difficult to diagnose and treat owing to lack of experience. ${ }^{1}$ Tumors consist of complex with malignant gland and spindle cell components. Sarcomatous portion of carcinosarcoma had diversities from $5 \%$ to $99 \%{ }^{2}$ Serum prostate-specific antigen (PSA) levels are low in comparison with extent of tumor size. Presenting symptoms of patients are com-

Received October 14, 2019, Revised October 25, 2019,

Accepted October 28, 2019

Corresponding Author: Young-Joo Kim

Department of Urology, Jeju National University College of Medicine, 15 Aran 13-gil, Jeju 63241, Korea

E-mail: kurology@jejunu.ac.kr

Tel: +82-64-717-1760, Fax: +82-64-717-1131

ORCID: https://orcid.org/0000-0001-5580-4338

- This work was supported by the 2019 education, research and student guidance grant funded by Jeju National University. monly urinary obstruction. Therapeutic modalities including radiation therapy (RT), ADT, chemotherapy (CTX) and surgery for carcinosarcoma of prostate had shown disappointing outcomes. It is hard to distinguish clearly between carcinosarcoma and other tumors in many cases, thus a diagnosis will be confirmed after biopsy. In this case, early biopsy is to help planning this rare case. We need to take into account carcinosarcoma which presented with low levels of serum PSA compared with aggravating cancer, in spite of the form of therapy.

\section{CASE REPORT}

An 80-year-old patient was diagnosed with prostate cancer 7 years ago. The PSA was $43.8 \mathrm{ng} / \mathrm{mL}$. Prostate biopsy was performed by guided transrectal ultrasonography. Histopathological examination of the specimen showed an

2019 (C) Copyright The Korean Urological Oncology Society and The Korean Prostate Society. All Rights Reserved. 
adenocarcinoma (Gleason score $3+3$ ). We performed magnetic resonance imaging, computed tomography (CT), whole-body bone scan, and chest X-ray (CXR). No metastatic lesions except bladder neck invasion were found (Fig.
1A). The patient underwent EBRT for prostate adenocarcinoma. At 1 year after EBRT, the level of PSA had risen gradually despite the fact that characters of prostate showed no changing on CT (Fig. 1B). Additional ADT had
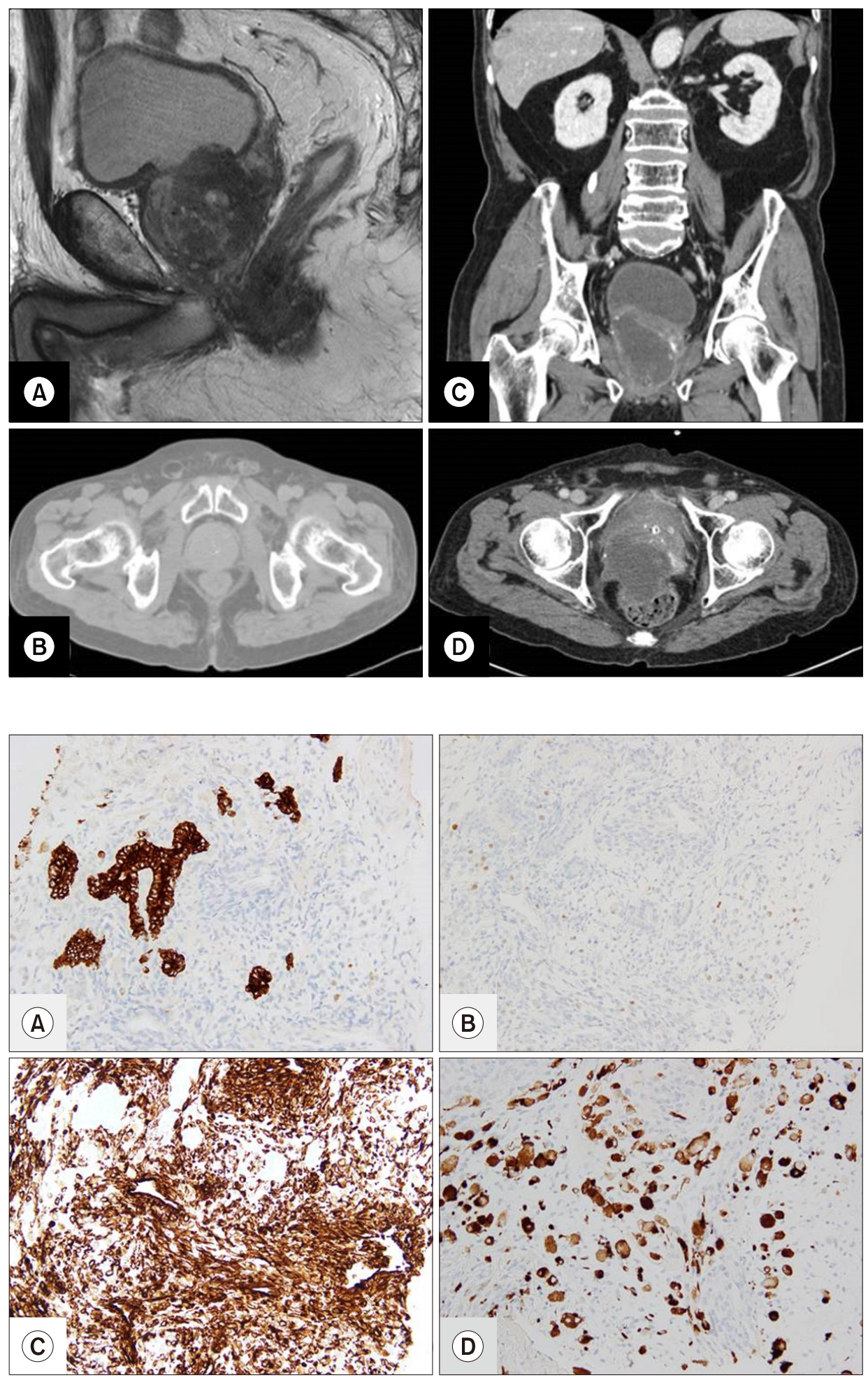

Fig. 1. (A) Magnetic resonance sagittal image shows that bladder neck invasion of prostatic adenocarcinoma on T2-weighted image. (B) Follow-up computed tomography shows a decreased prostate size at nadir state. Abdominal pelvic computed tomography anteriorposterior image $(\mathrm{C})$ and transverse image (D) show huge illdefined enhancing prostate.
Fig. 2. Immunohistochemical stain $(\times 200)$ : (A) spindle cells with marked atypical cells which are diffusely positive for cytokeratin, (B) spindle cells and polygonal large cells which are negative for p63, (C) spindle cells expressing vimentin, and (D) several spindle giant cells positively stained Desmin. 
been followed up 3 years. The serum PSA maintained level of the nadir was $0.002 \mathrm{ng} / \mathrm{mL}$. But, the patient did not visit hospital with a private matter during 3 years.

After 3 years, the patient visited Urology Department a complaining with urinary retention and rectal pain with constipation. On the physical examination, huge and hard rectal mass was palpable. The serum PSA was $3.51 \mathrm{ng} / \mathrm{mL}$. CXR showed multiple wide-spread variable-sized nodules in the entire both lung fields. Chest, abdomen and pelvis CT showed an 8-cm-sized rectal mass with poor enhancement and multiple lymph nodes metastasis (Fig. 1C, D). Colonoscopy guided biopsy was done to distinguish recurred prostate cancer and gastrointestinal stromal tumor. The current biopsy contains a minor component of high-grade prostatic acinar adenocarcinoma (10\%) and a major component of high-grade sarcoma (90\%). The high-grade sarcoma components show spindle cells with marked atypia and frequent mitoses and scattered bizarre polygonal large cells are positive for cytokeratin (Fig. 2A). Occasional spindle cells are negative for p63 represented for basal cell marker (Fig. 2B). They are diffusely positive for vimentin (Fig. 2C). The spindle cells and polygonal large cells are focally positive for desmin, smooth muscle actin and myoD1 but negative for ALK-1, CD34, CD117, PSA, and cytokeratin AE1/3 (Fig. 2D). The high-grade sarcomatous components show heterogenous sarcomatous elements consisting of predominantly rhabdomyosarcoma (60\%) and leiomyosarcoma (30\%). The high-grade acinar adenocarcinoma component is positive for cytokeratin AE1/3 and PSA (immunohistochemical stain, $\times 200$ ). This patient exhibited the short duration between initial presentation with adenocarcinoma and emergence of carcinosarcoma. The patient was scheduled for CTX as long as possible. Then, he was treated with docetaxel for one cycle. CTX was stopped because of rapid getting worse, and the patient died 5 months later.

\section{DISCUSSION}

The pathogenesis of prostatic carcinosarcoma is not clear and diverse analysis has been proposed for the various elements of carcinosarcoma. The prostatic carcinosarcomas interpreted the heterologous mix of carcinomatous (epithelial) and sarcomatous (mesenchymal) elements, and it could be related to previous $\mathrm{RT}$ or to $\mathrm{ADT}^{3}$ In our case, sarcomatous component was not found at the first biopsy. Carcinosarcoma was diagnosed with the second biopsy after 3 years of ADT with RT. Some reports content that RT and ADT may lead to transformation of carcinosarcoma. ${ }^{4}$ About half of carcinosarcoma cases, the first biopsies are proven acinar-typed adenocarcinoma. It was proposed the period for carcinosarcoma transformation from adenocarcinoma is averagely 3 years as seen in our case. ${ }^{5}$ Carcinosarcomas of prostate often are presented with low levels of serum PSA compared with adenocarcinoma, therefore serum PSA has low effects for the assessment of the sarcomatous element in carcinosarcoma, whereas it may be useful in assessing the progress of the adenocarcinoma component of the tumor. The epithelial component of carcinosarcoma of prostate stains shows positivity for cytokerarin and prostatic acid phosphatase. The sarcoma component of carcinosarcoma stains show negativity for PSA, epithelial membrane antigen, and keratin. ${ }^{6}$ It is hard to distinguish clearly between carcinosarcoma and other tumors in many diagnostic methods, thus a diagnosis will be confirmed after biopsy. The serum PSA remained low level after RT or ADT, nevertheless, the aggravating disease such as increasing tumor size or spreading metastasis was progress. In that case, it is good to planning for additional biopsy of the prostate. Early diagnosis and treatment may give a chance of improved prognosis in these patients. There is no standard option of treatment. There are various treatment modalities such as aggressive surgical treatments (radical prostatectomy or total pelvic exenteration) or EBRT or ADT or CTX. ADT would be preferred to treat the adenocarcinoma component of the prostatic cancer but would be useless to control the sarcomatoid component of the tumor. Aggressive surgery remains the recommendable therapy method. Early diagnosis and treatment may give a chance of improved prognosis in these patients. The most often use therapeutic modality has been an RT (43\%). Other modalities such as surgery (40\%), ADT (38\%), and CTX (14\%) have been used for the patients with prostatic carcinosarcoma in order of precedence. ${ }^{7}$ The sites of metastases were the lung, bone, lymph node, liver, brain, and peritoneum and so on. Most common site is lung. ${ }^{7}$ In cases of advanced disease supportive therapy would be required. Patients with severe obstruction of the rectum would require colostomy as seen in our case. The survival period was extremely disappoin- 
ting. The mean period was 7 months. ${ }^{8}$ Our patient was treated with CTX, but CTX was stopped because of rapid clinical aggravation, and the patient died 5 months later. Early rebiopsy of prostate in case of progressed disease despite of PSA maintenance after RT or ADT may help diagnose various carcinoma, and early aggressive treatment may help to improve prognosis.

\section{CONFLICT OF INTEREST}

The author claims no conflicts of interest.

\section{REFERENCES}

1. Açıkgöz O, Gazel E, Zengin NI, Kasap Y, Camtosun A, Yazıcıoğlu AH. Sarcomatoid carcinoma of the prostate. Case Rep Urol 2013;2013:631809.

2. Hansel DE, Herawi M, Montgomery E, Epstein JI. Spindle cell lesions of the adult prostate. Mod Pathol 2007;20:148-
58.

3. Hansel DE, Epstein JI. Sarcomatoid carcinoma of the prostate: a study of 42 cases. Am J Surg Pathol 2006;30:131621.

4. Venyo AK. Primary lymphoepithelioma-like carcinoma of the prostate gland: a review of the literature. Scientifica (Cairo) 2016;2016:1876218.

5. Ray ME, Wojno KJ, Goldstein NS, Olson KB, Shah RB, Cooney KA. Clonality of sarcomatous and carcinomatous elements in sarcomatoid carcinoma of the prostate. Urology 2006;67:423.e5-423.e8.

6. Wick MR, Young RH, Malvesta R, Beebe DS, Hansen JJ, Dehner LP. Prostatic carcinosarcomas. Clinical, histologic, and immunohistochemical data on two cases, with a review of the literature. Am J Clin Pathol 1989;92:131-9.

7. Fukawa T, Numata K, Yamanaka M, Miyamoto T, Kurokawa Y, Kanayama HO, et al. Prostatic carcinosarcoma: a case report and review of literature. Int J Urol 2003;10: 108-13.

8. Dundore PA, Cheville JC, Nascimento AG, Farrow GM, Bostwick DG. Carcinosarcoma of the prostate. Report of 21 cases. Cancer 1995;76:1035-42. 